\title{
A Study of Generalized Second Law of Thermodynamics in Magnetic Universe in the light of Non-Linear Electrodynamics
}

\author{
Tanwi Bandyopadhyay ${ }^{1 *}$ and Ujjal Debnath ${ }^{2 \dagger}$ \\ ${ }^{1}$ Department of Mathematics, Shri Shikshayatan College, 11, Lord Sinha Road, Kolkata-71, India. \\ ${ }^{2}$ Department of Mathematics, Bengal Engineering and Science University, Shibpur, Howrah-711103, India.
}

\begin{abstract}
In this work, we have considered the magnetic universe in non-linear electrodynamics. The Einstein's field equations for non-flat FRW model have been considered when the universe is filled with the matter and magnetic field only. We have discussed the validity of the generalized second law of thermodynamics of the magntic universe bounded by Hubble, apparent, particle and event horizons using Gibb's law and the first law of thermodynamics for interacting and non-interacting scenarios. It has been shown that the GSL is always satisfied for Hubble, apparent and particle horizons but for event horizon, the GSL is violated initially and satisfied at late stage of the universe.
\end{abstract}

\section{INTRODUCTION}

In present years the standard cosmological model based on Friedmann-Robertson-Walker (FRW) with Maxwell electrodynamics has got much attention and many interesting results are obtained. This leads to a cosmological singularity at a finite time in the past and result the energy density and curvature arbitrary large in the very early epoch [1]. This singularity breaks the laws of physics with mathematical inconsistency and physical incompleteness of any cosmological model. There are some proposals to handle this primordial singularity such as cosmological constant [2], non minimal couplings [3], modifications of geometric structure of space-time [4], non-equilibrium thermodynamics [5], Born-Infeld type nonlinear electromagnetic field [6] and so on. Very strong electromagnetic fields might help avoiding the occurrence of space-time singularities in the cosmological context [7]. The impact of very strong electromagnetic fields regarding the causality issue in cosmology is also of relevance [8].

Studying the equations of the non-linear electrodynamics (NLED) is an attractive subject of research in general relativity thanks to the fact that such quantum phenomena as vacuum polarization can be implemented in a classical model through their impact on the properties of the background space-time. Recently a new approach [9] has been taken to avoid the cosmic singularity through a nonlinear extension of the Maxwell electromagnetic theory. The associated Lagrangian and the resulting electrodynamics can theoretically be justified based on different arguments. Exact solutions of the Einsteins field equations coupled to NLED may hint at the relevance of the non-linear effects in strong gravitational and magnetic fields. For example, the nonlinear terms can be added to the standard Maxwell Lagrangian by imposing the existence of symmetries such as parity conservation, gauge invariance, Lorentz invariance etc [10] as well as by the introduction of first-order quantum corrections invariance to the Maxwell electrodynamics [11]. Another interesting feature can be viewed that an exact regular black hole solution has been recently obtained proposing Einstein-dual nonlinear electrodynamics [12]. Also the General Relativity (GR) coupled with NLED effects can explain the primordial inflation.

Since the discovery of black hole thermodynamics in 1970s, physicists have been speculating that there should be some relation between black hole thermodynamics and Einstein equations. In Einstein gravity, the evidence of this connection was first discovered in [13] by deriving the Einstein equation from the proportionality of entropy and horizon area together with the first law of thermodynamics $\delta Q=T d S$ in the Rindler spacetime. He assumed that this relation holds for all Rindler causal horizons through each space time point with $\delta Q$ and $T$ interpreted as the energy flux and temperature seen by an accelerated observer just inside the horizon. The horizon area (geometric quantity) of black hole is associated with its entropy (thermodynamical quantity), the surface gravity (geometric quantity) is related with its temperature (thermodynamical quantity) in black hole thermodynamics [14]. Verlinde [15] found that the Friedmann equation in a radiation dominated Friedmann-Robertson-Walker (FRW) universe can be written in an analogous form of the Cardy-Verlinde

\footnotetext{
*tanwib@gmail.com

$\dagger$ ujjal@iucaa.ernet.in
} 
formula, an entropy formula for a conformal field theory. The thermodynamics in de Sitter spacetime was first investigated by Gibbons and Hawking in [16].

The identity between Einstein equations and thermodynamical laws has been applied in the cosmological context considering universe as a thermodynamical system bounded by the apparent horizon. The first law of thermodynamics for the cosmological horizon is given by $-d E=T d S$, where $T=\frac{1}{2 \pi l}$ is the Hawking temperature, and $S=\frac{A}{4 G}$ is the entropy with $A=4 \pi l^{2}$ and $G$ as the cosmological horizon area and Newton constant respectively [17]. At the apparent horizon, the first law of thermodynamics (on the apparent horizon) is shown to be equivalent to Friedmann equations and the generalized second law of thermodynamics (GSLT) is obeyed at the horizon. In a spatially flat de Sitter spacetime, the event horizon and the apparent horizon of the Universe coincide and there is only one cosmological horizon. In the usual standard big bang model a cosmological event horizon does not exist. But for the accelerating universe dominated by dark energy, the cosmological event horizon separates from that of the apparent horizon. When the apparent horizon and the event horizon of the Universe are different, it was found that the first law and generalized second law (GSL) of thermodynamics hold on the apparent horizon, while they break down if one considers the event horizon [18]. On the basis of the well known correspondence between the Friedmann equation and the first law of thermodynamics of the apparent horizon, Gong et al [19] argued that the apparent horizon is the physical horizon in dealing with thermodynamics problems.

There are several studies in thermodynamics for dark energy filled universe on apparent and event horizons [20]. Setare and Shafei [21] showed that for the apparent horizon the first law is roughly respected for different epochs while the second law of thermodynamics is respected. Considering the interacting holographic model of dark energy to investigate the validity of the GSL of thermodynamics in a non-flat (closed) universe enclosed by the event horizon, Setare [22] found that generalized second law is respected for the special range of the deceleration parameter. The transition from quintessence to phantom dominated universe was considered and the conditions of the validity of GSL in transition was studied in [23]. In the reference [24], a Chaplygin gas dominated was considered and the GSL was investigated taking into account the existence of the observers event horizon in accelerated universes and it was concluded that for the initial stage of Chaplygin gas dominated expansion, the GSL of gravitational thermodynamics is fulfilled. Recently, the GSL of thermodynamics on Hubble, apparent, particle and event horizons have been extensively studied in [25].

In this work, we have briefly discussed the Maxwell's electrodynamics in linear and non-linear forms in section II. The energy density and pressure for non-linear electrodynamics have been written in magnetic universe only. The Einstein's field equations for non-flat FRW model have been considered if the universe is filled with the matter and magnetic field only. The interaction between matter and magnetic field have been considered in section III and some particular form of interaction term, we have found the solutions of magnetic field and the energy density of matter. In section IV, the validity of generalized second law of thermodynamics have been investigated on Hubble, apparent, particle and event horizons using Gibb's law and the first law of thermodynamics. Finally, we have made some concluding remarks.

\section{BRIEF OVERVIEW OF NON-LINEAR ELECTRODYNAMICS}

The Lagrangian density in Maxwell's electrodynamics can be written as [26]

$$
\mathcal{L}=-\frac{1}{4 \mu_{0}} F^{\mu \nu} F_{\mu \nu}=-\frac{1}{4 \mu_{0}} F
$$

where $F^{\mu \nu}$ is the electromagnetic field strength tensor and $\mu_{0}$ is the magnetic permeability. The canonical energy-momentum tensor is then given by

$$
T_{\mu \nu}=\frac{1}{\mu_{0}}\left(F_{\mu \alpha} F_{\nu}^{\alpha}+\frac{1}{4} F g_{\mu \nu}\right)
$$

The homogeneous, isotropic FRW model of the universe is given by 


$$
d s^{2}=d t^{2}-a^{2}(t)\left[\frac{d r^{2}}{1-k r^{2}}+r^{2}\left(d \theta^{2}+\sin ^{2} \theta d \phi^{2}\right)\right]
$$

Since the spatial section of FRW geometry are isotropic, electromagnetic fields can generate such a universe only if an averaging procedure is performed [27]. Applying standard spatial averaging process for electric field $E_{i}$ and magnetic field $B_{i}$, set

$$
<E_{i}>=0, \quad<B_{i}>=0, \quad<E_{i} E_{j}>=-\frac{1}{3} E^{2} g_{i j}, \quad<B_{i} B_{j}>=-\frac{1}{3} B^{2} g_{i j}, \quad<E_{i} B_{j}>=0 .
$$

So from equation (4) we get,

$$
<F_{\mu \alpha} F_{\nu}^{\alpha}>=\frac{2}{3}\left(\epsilon_{0} E^{2}+\frac{B^{2}}{\mu_{0}}\right) u_{\mu} u_{\nu}+\frac{1}{3}\left(\epsilon_{0} E^{2}-\frac{B^{2}}{\mu_{0}}\right) g_{\mu \nu}
$$

where $u_{\mu}$ is the fluid 4-velocity. Now comparing with the average value of energy momentum tensor

$$
<T_{\mu \nu}>=(\rho+p) u_{\mu} u_{\nu}-p g_{\mu \nu}
$$

the energy density and pressure have the forms

$$
\rho=\frac{1}{2}\left(\epsilon_{0} E^{2}+\frac{B^{2}}{\mu_{0}}\right), \quad p=\frac{1}{3} \rho
$$

This shows that the Maxwell's electrodynamics generates only the radiation type fluid in FRW universe.

Here we consider the generalization of Maxwell electro-magnetic Lagrangian up to the second order terms of the fields as [26]

$$
\mathcal{L}=-\frac{1}{4 \mu_{0}} F+\omega F^{2}+\eta F^{* 2}
$$

where $\omega$ and $\eta$ are arbitrary constants,

$$
F^{*} \equiv F_{\mu \nu}^{*} F^{\mu \nu}
$$

and $F_{\mu \nu}^{*}$ is the dual of $F_{\mu \nu}$. So the corresponding energy-momentum tensor for non-linear electro-magnetic theory has the form

$$
T_{\mu \nu}=-4 \frac{\partial \mathcal{L}}{\partial F} F_{\mu}^{\alpha} F_{\alpha \nu}+\left(\frac{\partial \mathcal{L}}{\partial F^{*}} F^{*}-\mathcal{L}\right) g_{\mu \nu}
$$

Now we consider the homogeneous electric field $E$ in plasma gives rise to an electric current of charged particles and then rapidly decays. So the squared magnetic field $B^{2}$ dominates over $E^{2}$, i.e., in this case, the average value $\left\langle E^{2}>\approx 0\right.$ and hence $F=2 B^{2}$. So $F$ is now only the function of magnetic field (vanishing electric component) and hence the FRW universe may be called the magnetic universe. Now, similar to above discussions, we get the energy density and the pressure for magnetic field have the forms [26]

$$
\rho_{B}=\frac{B^{2}}{2 \mu_{0}}\left(1-8 \mu_{0} \omega B^{2}\right)
$$

and 


$$
p_{B}=\frac{B^{2}}{6 \mu_{0}}\left(1-40 \mu_{0} \omega B^{2}\right)=\frac{1}{3} \rho_{B}-\frac{16}{3} \omega B^{4}
$$

It is to be noted that the density of the magnetic field must be positive, so the magnetic field $B$ must be satisfied $B<\frac{1}{2 \sqrt{2 \mu_{0} \omega}}$. Comparing (7) and (12), we say that $\frac{16}{3} \omega B^{4}$ is the correction term of the EOS for radiation in the generalization of Maxwell's electrodynamics.

The Einstein's field equations are given by

$$
H^{2}+\frac{k}{a^{2}}=\frac{8 \pi G}{3} \rho_{t o t a l}
$$

and

$$
\dot{H}-\frac{k}{a^{2}}=-4 \pi G\left(\rho_{\text {total }}+p_{\text {total }}\right)
$$

where

$$
\rho_{\text {total }}=\rho_{m}+\rho_{B}=\rho_{m}+\frac{B^{2}}{2 \mu_{0}}\left(1-8 \mu_{0} \omega B^{2}\right)
$$

and

$$
p_{\text {total }}=p_{m}+p_{B}=w_{m} \rho_{m}+\left(\frac{1}{3} \rho_{B}-\frac{16}{3} \omega B^{4}\right)
$$

where, $\rho_{m}$ and $p_{m}$ are energy density and pressure for matter obeys the equation of state $p_{m}=w_{m} \rho_{m}$.

Now the energy-conservation equation is

$$
\dot{\rho}_{\text {total }}+3 H\left(\rho_{\text {total }}+p_{\text {total }}\right)=0
$$

where $H=\frac{\dot{a}}{a}$ is the Hubble parameter.

\section{INTERACTION BETWEEN MATTER AND MAGNETIC FIELD}

Here we consider the interaction between matter and magnetic field. So the conservation equation (17) becomes

$$
\dot{\rho}_{m}+3 H\left(1+w_{m}\right) \rho_{m}=Q
$$

and

$$
\dot{\rho}_{B}+3 H\left(\rho_{B}+p_{B}\right)=-Q
$$

For simplicity of the calculation, we take the interaction component as

$$
Q=3 \delta \frac{B}{\mu_{0}}\left(1-16 \mu_{0} \omega B^{2}\right) H
$$


where $\delta$ is a small positive quantity.

Using the expressions of $\rho_{E}, p_{E}, \rho_{m}$ and $p_{m}$, the above two equations can be solved to obtain

$$
B=-\frac{3}{2} \delta+\frac{B_{0}}{a^{2}}, \quad B_{0} \text { being a constant }
$$

and

$$
\rho_{m}=\frac{3 \delta}{2 \mu_{0}}\left[-\frac{32 B_{0}^{2} \mu_{0} \omega}{3\left(w_{m}-1\right) a^{6}}+\frac{144 B_{0}^{2} \delta \mu_{0} \omega}{\left(3 w_{m}-1\right) a^{4}}-\frac{\delta\left(1-36 \delta^{2} \mu_{0} \omega\right)}{w_{m}+1}-\frac{2 B_{0}\left(108 \delta^{2} \mu_{0} \omega-1\right)}{\left(3 w_{m}+1\right) a^{2}}\right]+\rho_{0} a^{-3\left(1+w_{m}\right)}
$$

where $\rho_{0}$ is an integration constant. For the above solutions, we can verified that the interaction term $Q$ always decays with the evolution of the universe.

\section{GENERALIZED SECOND LAW OF THERMODYNAMICS}

In this section, the validity of the generalized second law of thermodynamics is studied. It states that, the sum of entropy of total matter enclosed by the horizon and the entropy of the horizon does not decrease with time. In the following, we consider Hubble, apparent, particle and event horizons. The variation of entropy inside the horizon will be calculated via Gibb's equation and the variation of entropy on the horizon will be

calculated using first law of thermodynamics. Hence we shall examine the validity of GSL of thermodynamics of the universe bounded by the above mentioned horizons.

\section{A. Hubble Horizon}

We know that radius of Hubble horizon [25],

$$
R_{H}=\frac{1}{H}
$$

Therefore

$$
\dot{R}_{H}=-\frac{\dot{H}}{H^{2}}=\frac{\frac{2 B^{2}}{\mu_{0}}\left(1-16 \mu_{0} \omega B^{2}\right)+3\left(1+w_{m}\right) \rho_{m}-\frac{3 k}{4 \pi G a^{2}}}{\frac{B^{2}}{\mu_{0}}\left(1-8 \mu_{0} \omega B^{2}\right)+2 \rho_{m}-\frac{3 k}{4 \pi G a^{2}}}
$$

Considering the net amount of energy crossing through the Hubble horizon in time $d t$ as [28]

$$
-d E=4 \pi R_{H}^{3} H\left(\rho_{\text {total }}+p_{\text {total }}\right) d t
$$

and assuming the validity of first law of thermodynamics on the Hubble horizon, i.e,

$$
-d E=T_{H} d S_{H}
$$

we have

$$
\frac{d S_{H}}{d t}=\frac{4 \pi R_{H}^{3} H}{T_{H}}\left[\frac{2 B^{2}}{3 \mu_{0}}\left(1-16 \mu_{0} \omega B^{2}\right)+\left(1+w_{m}\right) \rho_{m}\right]
$$

Again from the Gibbs' eqn [18, 24] 


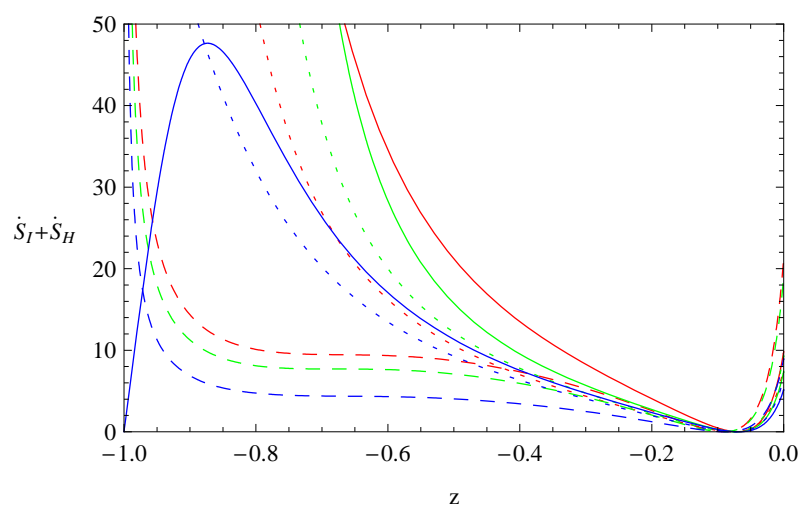

Fig.1

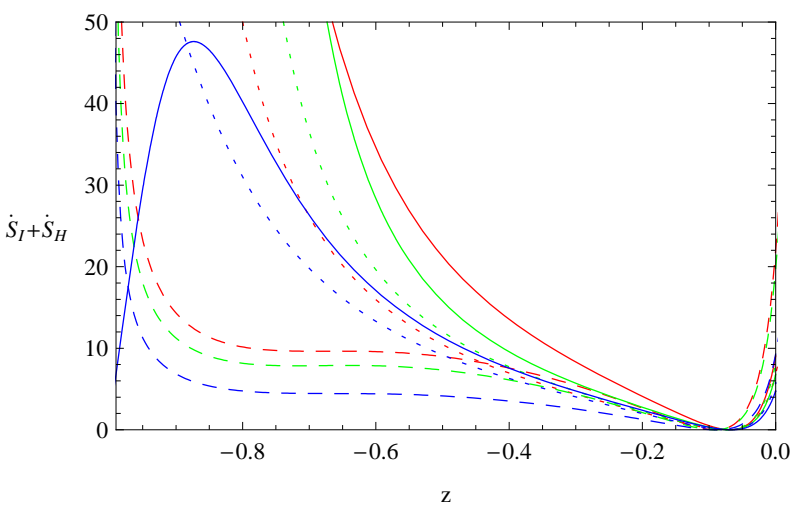

Fig.2

Figs. 1 and 2 represent rate of change of total entropy of Hubble horizon i.e., $\dot{S}_{H}+\dot{S}_{I}$ against redshift $z$ without and with interaction respectively for $w_{m}=1 / 3$ (solid line), $w_{m}=0$ (dotted line) and $w_{m}=-0.5$ (dashed line) and $k=0$ (red line), $k=+1$ (green line) and $k=-1$ (blue line).

$$
T_{H} d S_{I}=d E_{I}+p_{\text {total }} d V
$$

we have

$$
\frac{d S_{I}}{d t}=\frac{4 \pi R_{H}^{2}}{T_{H}}\left[\frac{2 B^{2}}{3 \mu_{0}}\left(1-16 \mu_{0} \omega B^{2}\right)+\left(1+w_{m}\right) \rho_{m}\right]\left(\dot{R}_{H}-1\right)
$$

where $S_{I}$ is the entropy inside the horizon, $V=\frac{4}{3} \pi R^{3}$ is the volume of the horizon universe, $E_{I}=\rho_{\text {total }} V$ is the internal energy and $T$ stands for the Hawking temperature.

From eqns (27) and (29), the rate of change of the total entropy becomes

$$
\frac{d}{d t}\left(S_{H}+S_{I}\right)=\frac{4 \pi R_{H}^{2}}{T_{H}}\left[\frac{2 B^{2}}{3 \mu_{0}}\left(1-16 \mu_{0} \omega B^{2}\right)+\left(1+w_{m}\right) \rho_{m}\right]\left[\frac{\frac{2 B^{2}}{\mu_{0}}\left(1-16 \mu_{0} \omega B^{2}\right)+3\left(1+w_{m}\right) \rho_{m}-\frac{3 k}{4 \pi G a^{2}}}{\frac{B^{2}}{\mu_{0}}\left(1-8 \mu_{0} \omega B^{2}\right)+2 \rho_{m}-\frac{3 k}{4 \pi G a^{2}}}\right]
$$

Here the expressions of $B$ and $\rho_{m}$ are given in (21) and (22). We plot the rate of change of total entropy of Hubble horizon i.e., $\dot{S}_{H}+\dot{S}_{I}$ against redshift $z$ in figures 1 and 2 , without $(\delta=0)$ and with interaction $(\delta=0.001)$ respectively for different matter components i.e., $w_{m}=1 / 3$ (solid line), $w_{m}=0$ (dotted line) and $w_{m}=-0.5$ (dashed line) and $k=0$ (red line), $k=+1$ (green line) and $k=-1$ (blue line). From these figures, we conclude that the GSL is always valid for Hubble horizon for non-interacting and interacting scenarios of the magnetic universe.

\section{B. Apparent Horizon}

We know that radius of apparent horizon [25],

$$
R_{A}=\frac{1}{\sqrt{H^{2}+\frac{k}{a^{2}}}}
$$

Therefore 


$$
\dot{R}_{A}=-\frac{H\left(\dot{H}-\frac{k}{a^{2}}\right)}{\left(H^{2}+\frac{k}{a^{2}}\right)^{3 / 2}}=\frac{\left[\frac{B^{2}}{\mu_{0}}\left(1-8 \mu_{0} \omega B^{2}\right)+2 \rho_{m}-\frac{3 k}{4 \pi G a^{2}}\right]^{1 / 2}\left[\frac{2 B^{2}}{\mu_{0}}\left(1-16 \mu_{0} \omega B^{2}\right)+3\left(1+w_{m}\right) \rho_{m}\right]}{\left[\frac{B^{2}}{\mu_{0}}\left(1-8 \mu_{0} \omega B^{2}\right)+2 \rho_{m}\right]^{3 / 2}}
$$

Considering the net amount of energy crossing through the apparent horizon in time $d t$ as [28]

$$
-d E=4 \pi R_{A}^{3} H\left(\rho_{\text {total }}+p_{\text {total }}\right) d t
$$

and assuming the validity of first law of thermodynamics on the apparent horizon, i.e,

$$
-d E=T_{A} d S_{A}
$$

we have

$$
\frac{d S_{A}}{d t}=\frac{4 \pi R_{A}^{3} H}{T_{A}}\left[\frac{2 B^{2}}{3 \mu_{0}}\left(1-16 \mu_{0} \omega B^{2}\right)+\left(1+w_{m}\right) \rho_{m}\right]
$$

Again from the Gibbs' eqn [18, 24]

$$
T_{A} d S_{I}=d E_{I}+p_{\text {total }} d V
$$

we have

$$
\frac{d S_{I}}{d t}=\frac{4 \pi R_{A}^{2}}{T_{A}}\left[\frac{2 B^{2}}{3 \mu_{0}}\left(1-16 \mu_{0} \omega B^{2}\right)+\left(1+w_{m}\right) \rho_{m}\right]\left(\dot{R}_{A}-H R_{A}\right)
$$

From eqns (35) and (37), the rate of change of the total entropy becomes

$$
\frac{d}{d t}\left(S_{A}+S_{I}\right)=\frac{12 \pi R_{A}^{2}}{T_{A}}\left[\frac{2 B^{2}}{3 \mu_{0}}\left(1-16 \mu_{0} \omega B^{2}\right)+\left(1+w_{m}\right) \rho_{m}\right]^{2} \frac{\left[\frac{B^{2}}{\mu_{0}}\left(1-8 \mu_{0} \omega B^{2}\right)+2 \rho_{m}-\frac{3 k}{4 \pi G a^{2}}\right]^{1 / 2}}{\left[\frac{B^{2}}{\mu_{0}}\left(1-8 \mu_{0} \omega B^{2}\right)+2 \rho_{m}\right]^{3 / 2}}
$$

Here the expressions of $\rho_{m}$ and $B$ are given in (21) and (22). We plot the rate of change of total entropy of apparent horizon i.e., $\dot{S}_{A}+\dot{S}_{I}$ against redshift $z$ in figures 3 and 4 , without $(\delta=0)$ and with interaction $(\delta=0.001)$ respectively for different matter components i.e., $w_{m}=1 / 3$ (solid line), $w_{m}=0$ (dotted line) and $w_{m}=-0.5$ (dashed line) and $k=0$ (red line), $k=+1$ (green line) and $k=-1$ (blue line). From these figures, we conclude that the GSL is always valid for apparent horizon for non-interacting and interacting scenarios of the magnetic universe.

\section{Particle Horizon}

The horizon radius is given by [25]

$$
R_{P}=a \int_{0}^{a} \frac{d a}{H a^{2}}
$$

The differential eqn of which can be written as

$$
\dot{R}_{P}=H R_{P}+1
$$




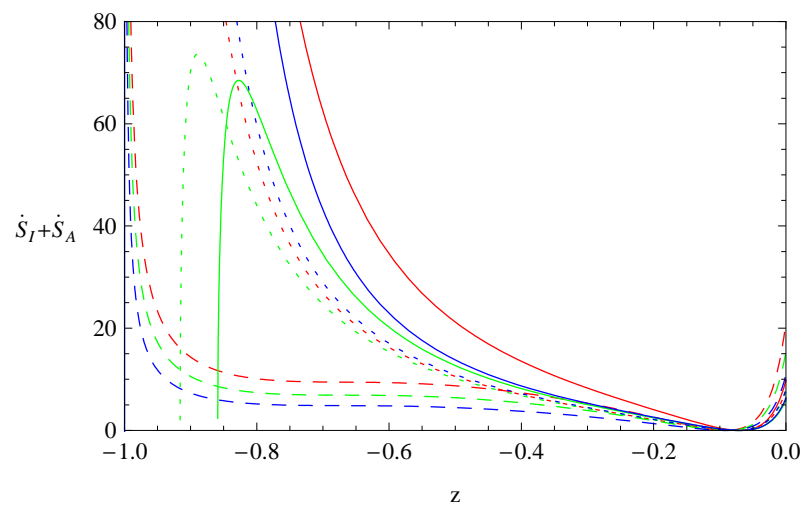

Fig.3

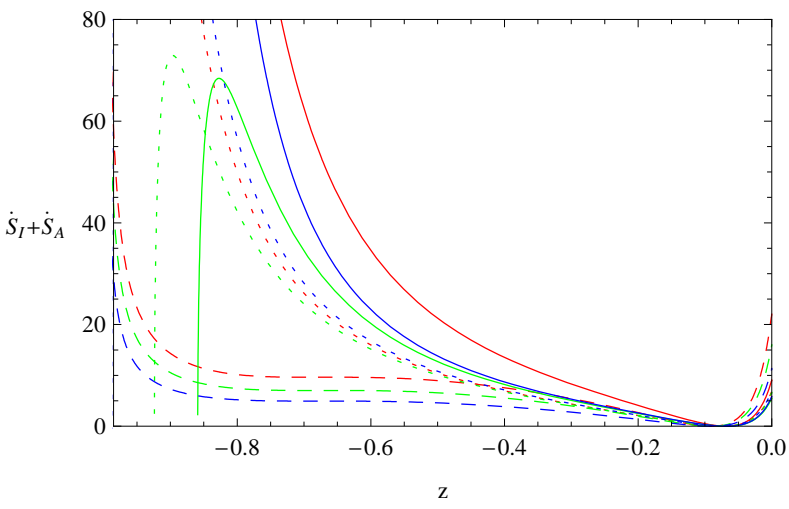

Fig.4

Figs. 3 and 4 represent rate of change of total entropy of apparent horizon i.e., $\dot{S}_{A}+\dot{S}_{I}$ against redshift $z$ without and with interaction respectively for $w_{m}=1 / 3$ (solid line), $w_{m}=0$ (dotted line) and $w_{m}=-0.5$ (dashed line) and $k=0$ (red line), $k=+1$ (green line) and $k=-1$ (blue line).

Considering the net amount of energy crossing through the particle horizon in time $d t$ as [28]

$$
-d E=4 \pi R_{P}^{3} H\left(\rho_{\text {total }}+p_{\text {total }}\right) d t
$$

and assuming the validity of first law of thermodynamics on the particle horizon, i.e,

$$
-d E=T_{P} d S_{P}
$$

we have

$$
\frac{d S_{P}}{d t}=\frac{4 \pi R_{P}^{3} H}{T_{P}}\left[\frac{2 B^{2}}{3 \mu_{0}}\left(1-16 \mu_{0} \omega B^{2}\right)+\left(1+w_{m}\right) \rho_{m}\right]
$$

Again from the Gibbs' eqn [18, 24]

$$
T_{P} d S_{I}=d E_{I}+p_{\text {total }} d V
$$

we have

$$
\frac{d S_{I}}{d t}=\frac{-4 \pi R_{P}^{2}}{T_{P}}\left[\frac{2 B^{2}}{3 \mu_{0}}\left(1-16 \mu_{0} \omega B^{2}\right)+\left(1+w_{m}\right) \rho_{m}\right]
$$

From eqns (43) and (45), the rate of change of the total entropy becomes

$$
\frac{d}{d t}\left(S_{P}+S_{I}\right)=\frac{4 \pi R_{P}^{2}}{T_{P}}\left[\frac{2 B^{2}}{3 \mu_{0}}\left(1-16 \mu_{0} \omega B^{2}\right)+\left(1+w_{m}\right) \rho_{m}\right]\left(H R_{P}+1\right)
$$

We plot the rate of change of total entropy of particle horizon i.e., $\dot{S}_{P}+\dot{S}_{I}$ against redshift $z$ in figures 5 and 6 , without $(\delta=0)$ and with interaction $(\delta=0.001)$ respectively for different matter components i.e., $w_{m}=1 / 3$ (solid line), $w_{m}=0$ (dotted line) and $w_{m}=-0.5$ (dashed line) and $k=0$ (red line), $k=+1$ (green line) and $k=-1$ (blue line). From these figures, we conclude that the GSL is always valid for particle horizon for non-interacting and interacting scenarios of the magnetic universe. 


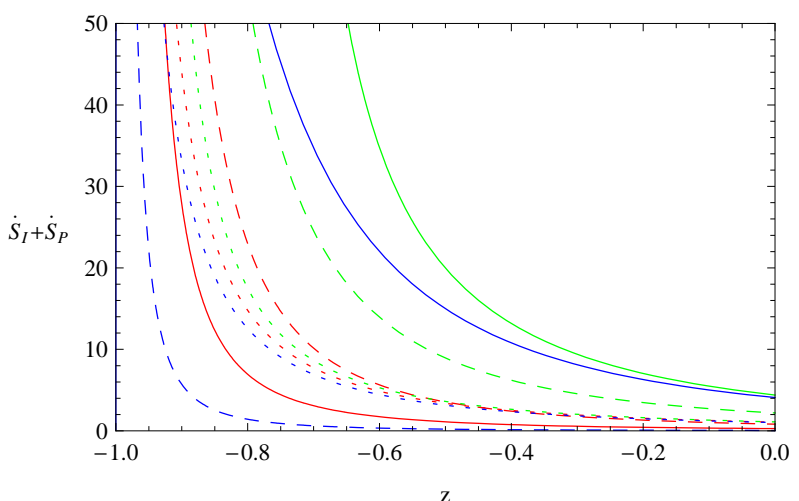

Fig.5

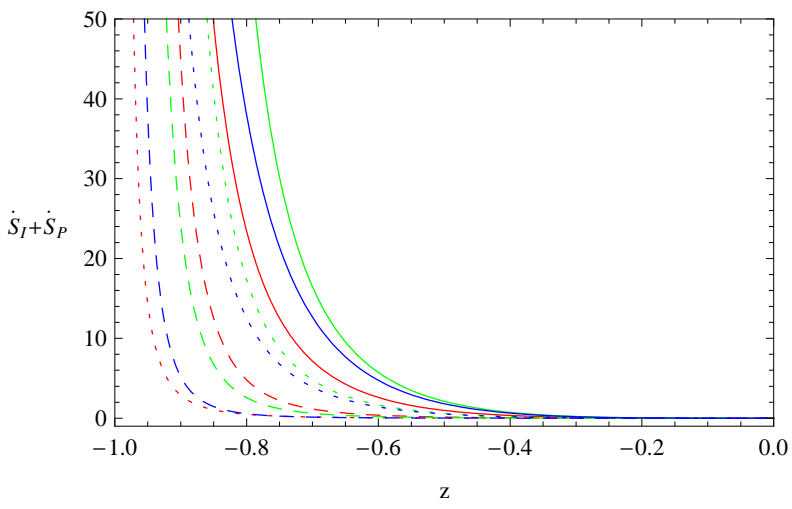

Fig.6

Figs. 5 and 6 represent rate of change of total entropy of particle horizon i.e., $\dot{S}_{P}+\dot{S}_{I}$ against redshift $z$ without and with interaction respectively for $w_{m}=1 / 3$ (solid line), $w_{m}=0$ (dotted line) and $w_{m}=-0.5$ (dashed line) and $k=0$ (red line), $k=+1$ (green line) and $k=-1$ (blue line).

\section{Event Horizon}

The horizon radius is given by [25]

$$
R_{E}=a \int_{a}^{\infty} \frac{d a}{H a^{2}}
$$

The differential eqn of which can be written as

$$
\dot{R}_{E}=H R_{E}-1
$$

Considering the net amount of energy crossing through the event horizon in time $d t$ as [28]

$$
-d E=4 \pi R_{E}^{3} H\left(\rho_{\text {total }}+p_{\text {total }}\right) d t
$$

and assuming the validity of first law of thermodynamics on the event horizon, i.e,

$$
-d E=T_{E} d S_{E}
$$

we have

$$
\frac{d S_{E}}{d t}=\frac{4 \pi R_{E}^{3} H}{T_{E}}\left[\frac{2 B^{2}}{3 \mu_{0}}\left(1-16 \mu_{0} \omega B^{2}\right)+\left(1+w_{m}\right) \rho_{m}\right]
$$

Again from the Gibbs' eqn [18, 24]

$$
T_{E} d S_{I}=d E_{I}+p_{t o t a l} d V
$$

we have

$$
\frac{d S_{I}}{d t}=\frac{-4 \pi R_{E}^{2}}{T_{E}}\left[\frac{2 B^{2}}{3 \mu_{0}}\left(1-16 \mu_{0} \omega B^{2}\right)+\left(1+w_{m}\right) \rho_{m}\right]
$$




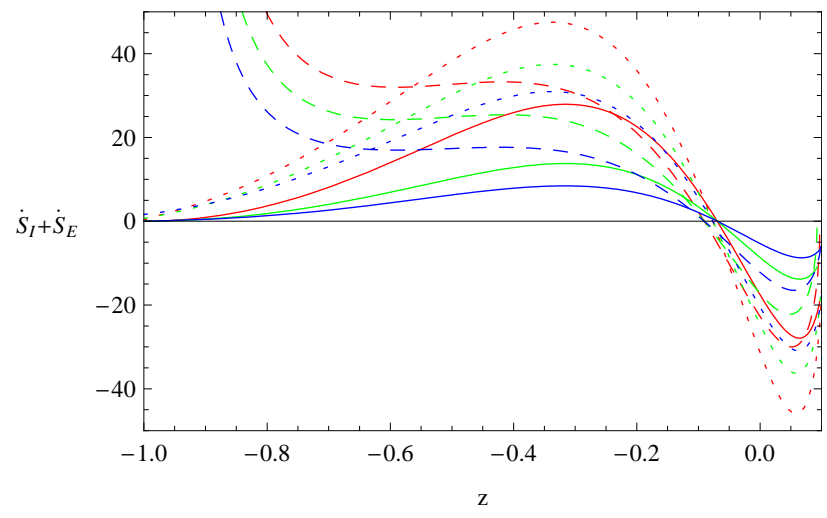

Fig.7

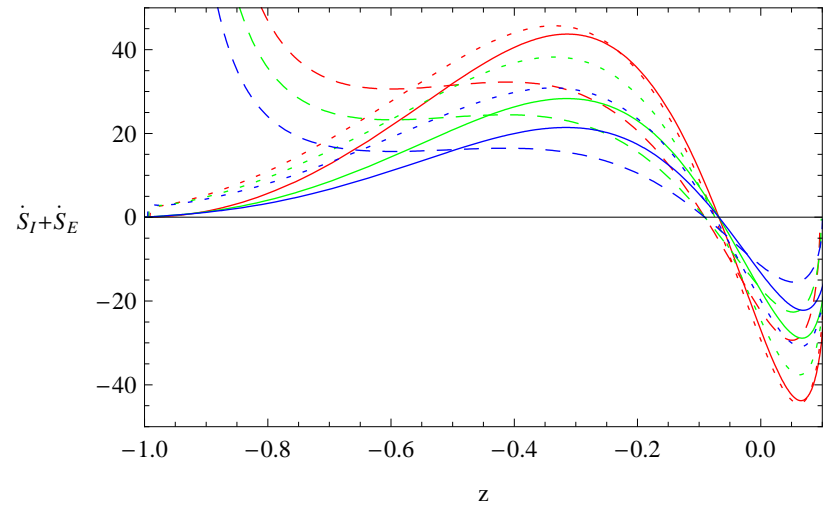

Fig.8

Figs. 7 and 8 represent rate of change of total entropy of event horizon i.e., $\dot{S}_{E}+\dot{S}_{I}$ against redshift $z$ without and with interaction respectively for $w_{m}=1 / 3$ (solid line), $w_{m}=0$ (dotted line) and $w_{m}=-0.5$ (dashed line) and $k=0$ (red line), $k=+1$ (green line) and $k=-1$ (blue line).

From eqns (51) and (53), the rate of change of the total entropy becomes

$$
\frac{d}{d t}\left(S_{E}+S_{I}\right)=\frac{4 \pi R_{E}^{2}}{T_{E}}\left[\frac{2 B^{2}}{3 \mu_{0}}\left(1-16 \mu_{0} \omega B^{2}\right)+\left(1+w_{m}\right) \rho_{m}\right]\left(H R_{E}-1\right)
$$

We plot the rate of change of total entropy of event horizon i.e., $\dot{S}_{E}+\dot{S}_{I}$ against redshift $z$ in figures 7 and 8 , without $(\delta=0)$ and with interaction $(\delta=0.001)$ respectively for different matter components i.e., $w_{m}=1 / 3$ (solid line), $w_{m}=0$ (dotted line) and $w_{m}=-0.5$ (dashed line) and $k=0$ (red line), $k=+1$ (green line) and $k=-1$ (blue line). From these figures, we see that the rate of change of total entropy is negative level upto certain stage (about $z>-0.1$ ) and positive level after that stage for non-interacting and interacting scenarios of the magnetic universe bounded by event horizon. So we conclude that GSL is not satisfied upto certain stage and after this stage, it is always satisfied for magnetic universe bounded by event horizon.

\section{DISCUSSIONS}

In this work, we have briefly discussed the Maxwell's electrodynamics in linear and non-linear forms. The energy density and pressure for non-linear electrodynamics have been written in magnetic universe only. The Einstein's field equations for non-flat FRW model have been considered when the universe is filled with the matter and magnetic field only. The interaction between matter and magnetic field have been considered and some particular form of interaction term, we have found the solutions of magnetic field and the energy density of matter. The interaction term is always decreases with the time.

In the present work, our endeavor was to investigate the validity of the generalized second law of thermodynamics of the magnetic universe bounded by the Hubble, apparent, particle and event horizons. It states that, the sum of entropy of total matter enclosed by the horizon and the entropy of the horizon does not decrease with time. The variation of entropy inside the horizon have been calculated via Gibb's equation and the variation of entropy on the horizon have been calculated using first law of thermodynamics. We have investigated the generalized second law of the universe bounded by the above mentioned horizons.

In figures $1-8$, we have drawn the variation of total entropy on Hubble, apparent, particle and event horizons against redshift $z$ for non-interacting $(\delta=0)$ and interacting $(\delta=0.001)$ scenarios of magnetic universe for $k=0, \pm 1$ and $w_{m}=0,1 / 3,-0.5$. From figures $1-6$, we have seen that the rate of change of total entropy is always positive level for redshift $z$ decreases for interacting and non-interacting scenarios of the magnetic universe bounded by Hubble, apparent and particle horizons. But figures $7-8$ show the rate of change of total entropy 
is negative level upto certain stage (about $z>-0.1$ ) and positive level after that stage for non-interacting and interacting scenarios of the magnetic universe bounded by event horizon. So these imply, the GSL is always satisfied for magnetic universe bounded by Hubble, apparent and particle horizons. Also GSL is not satis-

fied upto certain stage and after this stage, it is always satisfied for magnetic universe bounded by event horizon.

\section{Acknowledgement:}

The authors are thankful to IUCAA, Pune, for their warm hospitality and excellent research facilities where most of the work has been done during a visit under the Associateship Programme. Also TB wants to thank UGC for providing with a project on thermodynamics.

\section{References:}

[1] E. W. Kolb and M. S. Turner, Addison-Wesley, Redwood City, CA (1990).

[2] W. de Sitter, Proc. K. Ned. Akad. Wet., 191217 (1917).

[3] M. Novello and J. M. Salim, Phys. Rev. D 20377 (1979).

[4] M. Novello et al, IJMPA 1641 (1993).

[5] G. L. Murphy, Phys. Rev. D 84231 (1973).

[6] R. Garca-Salcedo and N. Breton, IJMPA 154341 (2000).

[7] R. Garca-Salcedo and N. Breton, Class. Quant. Grav. 224783 (2005).

[8] M. Novello, Int. J. Mod. Phys. A 202421 (2005).

[9] V. A. De Lorenci, R. Klippert, M. Novello, and J. M. Salim, Phys. Rev. D 65063501 (2002).

[10] M. Novello, L. A. R. Oliveira and J. M. Salim, Class. Quantum Grav. 131089 (1996); G. Munõz, Am. J. Phys. 641285 (1996).

[11] W. Heisenberg and H. Euler, Z. Phys. 98714 (1936); J. Schwinger, Phys. Rev. 82664 (1951).

[12] H. Salazar, A. Garcia and J. Plebański, J. Math. Phys. 282171 (1987); E. Ayoń-Beato and A. Garcia, Phys. Rev. Lett. 805056 (1998).

[13] T. Jacobson, Phys. Rev. Lett. 751260 (1995).

[14] J. D. Bekenstein, Phys. Rev. D 72333 (1973); S. W. Hawking, Commun. Math. Phys. 43199 (1975); J. M. Bardeen, B. Carter and S. W. Hawking, Commun. Math. Phys. 31161 (1973).

[15] E. Verlinde, hep-th/0008140.

[16] G. W. Gibbons and S. W. Hawking, Phys. Rev. D 152738 (1977).

[17] R. G. Cai and S. P. Kim, JHEP 02050 (2005); Y. Gong and A. Wang, Phys. Rev. Lett. 99211301 (2007); R-G. Cai and L-M. Cao, Phys. Rev. D 75064008 (2007); X-H. Ge, Phys. Lett. B 65149 (2007); 6. M. Akbar and R-G. Cai, Phys. Lett. B 6357 (2006).

[18] B. Wang, Y. G. Gong and E. Abdalla, Phys. Rev. D 74083520 (2006).

[19] Y. Gong, B. Wang and A. Wang, JCAP 01024 (2007). 
[20] L. N. Granda and A. Oliveros, Phys. Lett. B 669275 (2008); Y. Gong, B. Wang and A. Wang, JCAP 01024 (2007); T. Padmanabhan, Class. Quantum Grav. 195387 (2002); R. -G. Cai and N. Ohta, Phys. Rev. D 81 084061; R. G. Cai and L. -M. Cao, Nucl. Phys. B 785135 (2007); M. Akbar and R. -G. Cai, Phys. Lett. B 6357 (2006); R. -G. Cai, L. -M. Cao, Y. -P. Hu and S. P. Kim, Phys. Rev. D 78124012 (2008).

[21] M. R. Setare and S. Shafei, JCAP 09011 (2006).

[22] M. R. Setare, JCAP 01023 (2007).

[23] M. R. Setare, Phys. Lett. B 641130 (2006).

[24] G. Izquierdo and D. Pavon, Phys. Lett. B 633420 (2006).

[25] S. Bhattacharya and U. Debnath, arXiv:1006.2600 [gr-qc]; A. Das, S. Chattopadhyay and U. Debnath, arXiv:1104.2378 [physics.gen-ph].

[26] C. S. Camara, M. R. de Garcia Maia, J. C. Carvalho and J. A. S. Lima, Phys. Rev. D 69123504 (2004).

[27] R. C. Tolman and P. Ehrenfest, Phys. Rev. 36, 1791 (1930); M. Hindmarsh and A. Everett, Phys. Rev. D 58, 103505 (1998).

[28] R. Bousso, Phys. Rev. D 71064024 (2005). 